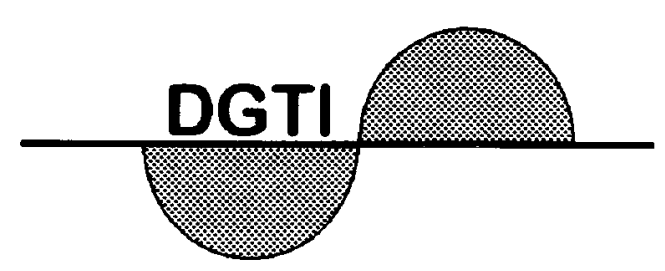

\section{Deutsche Gesellschaft für Transfusionsmedizin und Immunhämatologie}

Geschäftsstelle: Prof. Dr. R einhold E ckstein, A bteilung für Transfusionsmedizin und $\mathrm{H}$ ämostaseologie, U niversitätsklinikum E rlangen/N ürnberg, K rankenhausstraße 12, D -91054 E rlangen Tel. +49 9131 85-6346, Fax -6987

\section{J ahresbericht der Sektion «Präparative und Therapeutische Hämapherese»}

D ie Sektion «Präparative und Therapeutische $\mathrm{H}$ ämapherese» hat sich mit der standardisierten Herstellung von Granulozytenkonzentraten von G -CSF-konditionierten B lutspendern, der zentralen $D$ atenerfassung zur B lutstammzellapherese und Transplantation sowie der Qualitätssicherung in der $\mathrm{Häm}$ apherese befaßt.

Für die Herstellung von G ranulozytaphereseprodukten wurde ein Informationsschreiben und eine Einverständniserklärung für G-CSF-konditionierte Blutspender erarbeitet. Des weiteren wurden von der A rbeitsgruppe «G ranulozytapherese» E mpfehlungen zur Spendereignungsuntersuchung für die G ranulozytapherese, die Spendefrequenz, die G -CSF D osis, die G ranulozytenpräparation und -transfusion erstellt.
E ine Sektionsstudie zur Vorbehandlung mit rekombinantem G-CSF für die präparative Granulozytapherese wurde zwischenzeitlich initiiert. Inhalt der zweijährigen Studie ist die Ermittlung der Verträglichkeit von rekombinantem G-CSF bei der Spenderkonditionierung und der E influß von G-CSF auf die $\mathrm{G}$ ranulozytenpräparation.

Zur zentralen Datenerfassung zur Blutstammzellapherese und Transplantation wurde eine Fragebogenaktion bei den deutschen transfusionsmedizinischen E inrichtungen durchgeführt. Vor der Implementierung einer zentralen D atenerfassung soll zunächst noch eine mögliche Kooperation mit der $D$ eutschen A rbeitsgemeinschaft für K nochenmark- und Blutstammzelltransplantation (DA G -K BT) abgekärt werden.

B ei der Q ualitätssicherung in der $\mathrm{H}$ ämapherese beschäftigte sich die Sektion mit dem Stand der Fortbildung des Personals in Plasmaphereseeinrichtungen in Deutschland sowie mit einem Firmen-Q ualitätssicherungsprogramm.

Sektionsmitglieder erarbeiteten in den DGTI-ad-hoc-Kommissionen «Blutstammzellapherese», «Präparative L eukound Thrombozytapherese» und «Therapeutische Hämapherese» Durchfürungsempfehlungen zu den jeweiligen $A$ pheresearten. Teil I (Blutstammzellapherese) und Teil II (Präparative Leuko- und Thrombozytapherese) sind zwischenzeitlich in den Gesellschaftsmitteilungen der INFU SIONSTHE RAPIE UND TRANSFUSIONSMEDIZIN publiziert worden.

E rstmalig wurde während des Kongresses in B ern ein O peratorseminar mit dem Schwerpunktthema «Blutstammzellapherese» abgehalten. Ferner werden zunehmend regionale 0 peratorseminare angeboten.

\section{Dr. R. M oog}

Institut für Transfusionsmedizin

U niversitätsklinikum E ssen

H ufelandstraße 55, D-45122 E ssen

Tel. +49 201 723-1558, Fax -5945 